\title{
Killing of Serratia marcescens biofilms with chloramphenicol
}

\author{
Christopher Ray, Anukul T. Shenoy, Carlos J. Orihuela and Norberto González-Juarbe* (i)
}

\begin{abstract}
Serratia marcescens is a Gram-negative bacterium with proven resistance to multiple antibiotics and causative of catheter-associated infections. Bacterial colonization of catheters mainly involves the formation of biofilm. The objectives of this study were to explore the susceptibility of $S$. marcescens biofilms to high doses of common antibiotics and non-antimicrobial agents. Biofilms formed by a clinical isolate of $S$. marcescens were treated with ceftriaxone, kanamycin, gentamicin, and chloramphenicol at doses corresponding to 10, 100 and 1000 times their planktonic minimum inhibitory concentration. In addition, biofilms were also treated with chemical compounds such as polysorbate-80 and ursolic acid. S. marcescens demonstrated susceptibility to ceftriaxone, kanamycin, gentamicin, and chloramphenicol in its planktonic form, however, only chloramphenicol reduced both biofilm biomass and biofilm viability. Polysorbate-80 and ursolic acid had minimal to no effect on either planktonic and biofilm grown $\mathrm{S}$. marcescens. Our results suggest that supratherapeutic doses of chloramphenicol can be used effectively against established $\mathrm{S}$. marcescens biofilms.
\end{abstract}

Keywords: Serratia marcescens, Biofilm, Antibiotics, Chloramphenicol

\section{Background}

Serratia marcescens is a Gram-negative bacterium that causes infections in plants, insects, and animals, including humans [1]. Initially believed to be non-pathogenic, S. marcescens is currently known to cause ocular, pulmonary, urinary tract, and blood-stream infections $[1$, 2]. Recent reports implicate this pathogen in $2.5-7.7 \%$ of catheter-associated infections such as central lineassociated bloodstream infections (CLABSI) and catheter-associated urinary tract infections [3-5]. In the United States alone, an estimated 80,000 catheter-associated bloodstream infections occur every year with costs up to $\$ 56,000$ per episode [6]. Bacterial colonization of catheters confers a survival advantage and leads to the formation of cooperative bacterial communities known as "biofilms" [7]. Bacterial biofilms are complex surface-adhered communities of viable and dead bacteria, encased within an extracellular matrix composed of polysaccharide, protein and extracellular DNA [8]. In

*Correspondence: norberto@uab.edu Department of Microbiology, The University of Alabama at Birmingham, Birmingham, AL, USA addition, bacteria adhere to host's epithelial cells through formation of biofilm $[9,10]$. The ability of $S$. marcescens to form biofilms contributes to its pathogenicity $[1,11]$. Bacteria in biofilms also exhibit increased resistance to antimicrobial agents for reasons such as resistance to antibiotic penetration and metabolic changes such as slower growth rates $[7,12]$.

One current treatment used for salvaging biofilm-colonized catheters implicated in CLABSI is the application of antibiotic lock therapy (ALT). During ALT, supratherapeutic concentrations of antibiotic solutions are locked in the affected catheter for an extended amount of time [13, 14]. However, to our knowledge, the effect of ALT on S. marcescens biofilms has not yet been studied extensively in vivo nor in vitro. Another biofilm-control strategy centers on chemicals with no antimicrobial activity, which affects biofilm formation and adhesion by alteration of the bacterial microenvironment [12]. Recent reports have demonstrated the efficacy of polysorbate- 80 (PS80) a nonionic surfactant, in reducing the biofilm mass of Escherichia coli, Stenotrophomonas maltophilia, Listeria monocytogenes, and Pseudomonas fluorescens [15-17]. PS80 is a solubilizing and emulsifying agent 
used in ointments, lotions, soaps as well as in medical preparations such as vitamin oils and anticancer agents [15]. In addition to PS80, ursolic acid (UA) is a plantderived agent shown to reduce Actinomyces viscosus, Streptococcus mutants, Vibrio harveyi, and Pseudomonas aeruginosa biofilm mass $[18,19]$. Herein, we determined the effectiveness of high-dose antibiotic, PS80 and UA therapies on in vitro biofilms formed by a clinical isolate of $S$. marcescens.

\section{Methods}

\section{Planktonic bacterial growth}

The tetracycline-resistant $S$. marcescens clinical isolate UT-383 was obtained from Dr. Jan Patterson (Division of Infectious Disease, Department of Medicine, The University of Texas Health Sciences Center at San Antonio) [2]. S. marcescens was grown on Luria-Bertani (LB) agar plates (LB-Lennox formulation) and incubated overnight at $37^{\circ} \mathrm{C}$. A single colony was transferred to LB broth and incubated overnight at $37^{\circ} \mathrm{C}$. The culture was then back diluted in fresh $\mathrm{LB}$ broth and incubated for $3 \mathrm{~h}$ at $37^{\circ} \mathrm{C}$ to an optical density $\left(\mathrm{OD}_{621}\right)$ of $0.5 \mathrm{~nm}$. To establish the minimum inhibitory concentration (MIC) of ceftriaxone, kanamycin, gentamicin and chloramphenicol on S. marcescens. Planktonic grown bacteria were exposed to the latter antibiotics, plates were incubated overnight, and MIC was determined as the lowest concentration of antibiotics with no growth detected by spectrophotometry at $\mathrm{OD}_{621}$.

\section{Biofilm growth and quantification}

Biofilm growth was initiated by inoculating $4 \mathrm{ml}$ of LB broth with $1 \times 10^{5} \mathrm{CFU}$ of $S$. marcescens using a 6-well polystyrene plate (Corning Inc., Corning NY). Cultures were grown overnight at $37{ }^{\circ} \mathrm{C} .3 \mathrm{ml}$ of media were then gently aspirated and replaced with $1 \mathrm{ml}$ of LB and $2 \mathrm{ml}$ of Mueller-Hinton broth (MH) (control) or $\mathrm{MH}$-based antibiotic solutions (ceftriaxone: $0.005,0.05,0.5 \mathrm{mg} / \mathrm{ml}$; kanamycin: $0.625,6.25,62.5 \mathrm{mg} / \mathrm{ml}$; gentamicin: 0.08 , $0.80,8.0 \mathrm{mg} / \mathrm{ml}$; and chloramphenicol: $0.08,0.80 \mathrm{mg} / \mathrm{ml}$ ). Solutions of PS80 were prepared to yield final concentrations of $1,0.1$, and $0.01 \%$, and UA to yield a final concentration of $0.03 \mathrm{mg} / \mathrm{ml}$. Biofilms were then returned to the incubator overnight. The media on each well was then aspirated and biofilms stained with $1 \%$ crystal violet for $10 \mathrm{~min}$, washed twice with $2 \mathrm{ml}$ of $1 \times$ PBS, dried, and photographed. A representative image of each biofilm was taken with a Leica LMD6 inverted microscope with a DFC3000G-1.3-megapixel monochrome camera (Leica Biosystems, Buffalo Grove, IL). Bacterial biofilms were then solubilized with $2 \mathrm{ml}$ of $95 \%$ ethanol, rocked for $30 \mathrm{~min}$ and the optical density at $540 \mathrm{~nm}$ of a 1:10 ethanol dilution was measured using an iMark Absorbance
Microplate Reader (Bio-Rad Laboratories, Hercules, CA). Controls of each antibiotic were incubated sterile overnight, stained, solubilized, and read in the same manner to account for background staining from each concentration of the solution.

Absorbance values were calculated by subtracting the background stain from each well. Values were then normalized to 1 by dividing by the mean growth of the bacteria-only controls of each experiment (which were calculated by subtracting the mean of all media-only wells from each bacteria-only control). A total of three experiments were carried out with ceftriaxone, kanamycin, and gentamicin (5 total replicates), while four experiments were carried out with chloramphenicol, PS80, and UA (7 total replicates).

\section{Viability of biofilms treated with chloramphenicol or PS80} After $24 \mathrm{~h}$ of growth, biofilms were treated with chloramphenicol or PS80 and incubated for an extra $24 \mathrm{~h}$ at $37^{\circ} \mathrm{C}$. Biofilms were then washed gently with $2 \mathrm{ml}$ of sterile PBS to dislodge remaining planktonic bacteria, and dried overnight at room temperature. Subsequently, $1 \mathrm{ml}$ of sterile PBS was added to each well and biofilms removed by scraping. The supernatant was serially diluted in PBS, then $100 \mu \mathrm{l}$ of each dilution was plated on individual blood agar petri dishes and incubated overnight to quantify the viable colony-forming units in the biofilm $[2,20]$.

\section{Antibiotics and other chemicals}

Antibiotic solutions were made in Mueller-Hinton broth (MH). Ceftriaxone (Sigma-Aldrich, St. Louis MO), kanamycin (Sigma-Aldrich), gentamicin (Gibco, Grand Island NY), and chloramphenicol (Sigma-Aldrich). Polysorbate-80 (Fisher Scientific, Fair Lawn NJ) and ursolic acid (Sigma-Aldrich).

\section{Statistical analysis}

Each set of experiments was then analyzed separately using Kruskal-Wallis ANOVA with multiple comparisons on GraphPad Prism version 7.0a (La Jolla CA).

\section{Results \\ High concentrations of chloramphenicol reduce biofilm growth of $S$. marcescens}

To test the susceptibility $S$. marcescens to multiple antibiotics, we co-cultured the latter in the presence of ceftriaxone, kanamycin, gentamicin, chloramphenicol, tetracycline, and erythromycin. Planktonic S. marcescens demonstrated susceptibility to all tested antibiotics except tetracycline and erythromycin at various doses (Additional file 1: Table S1). We then tested the ability of the antibiotics mentioned above to reduce $S$. marcescens 
biofilm biomass in vitro. S. marcescens biofilms were treated with ceftriaxone, kanamycin, gentamicin, and chloramphenicol at 10, 100, and 1000 times the planktonic minimum inhibitory concentration (MIC, see Additional file 2: Table S2). Biofilm biomass showed no change after treatment with $0.005,0.05,0.5 \mathrm{mg} / \mathrm{ml}$ of ceftriaxone (Fig. 1). At the lowest tested concentration of kanamycin $(0.625 \mathrm{mg} / \mathrm{ml})$ biofilm biomass was reduced. However, at $62.5 \mathrm{mg} / \mathrm{ml}$ of kanamycin, no change in biofilm formation was observed. Similar results also occurred when gentamicin was used (Fig. 1). These findings suggest a lack of dose-dependent effect in the reduction of S. marcescens biofilm biomass by kanamycin, gentamicin, and ceftriaxone; and raise the possibility that antibiotics promote biofilm formation.

Chloramphenicol is a last resort antibiotic used to treat infections such as tetracycline-resistant cholera [21] or brain abscesses caused by bacteria [22-24]. To determine if high doses of chloramphenicol could reduce biofilm biomass we co-cultured $S$. marcescens with 0.08 and $0.80 \mathrm{mg} / \mathrm{ml}$ of the latter antibiotic. Biofilm mass as determined by crystal violet staining showed a significant decrease at both $0.08 \mathrm{mg} / \mathrm{ml}(\mathrm{P}=0.0003)$ and $0.80 \mathrm{mg} /$ $\mathrm{ml}(\mathrm{P}<0.0001)$ of chloramphenicol, with a greater reduction at $0.80 \mathrm{mg} / \mathrm{ml}$ indicating dose-dependence (Fig. 2). Finally, biofilms treated with either PS80 or UA had a minimal but not significant change in biomass (Fig. 2). Collectively, these results suggest that antibiotics commonly used for ALT are not sufficient to reduce biomass of biofilms formed by a clinical isolate of $S$. marcescens except for chloramphenicol.

\section{PS80 1\% and high dose chloramphenicol lessen the viability of $S$. marcescens biofilms}

Quantification of viable bacteria from in vitro biofilms treated with both PS80 and chloramphenicol showed a significant reduction in the number of colony-forming units recovered after treatment (Fig. 3). These results suggest that PS80 and chloramphenicol could not only decrease $S$. marcescens biofilm biomass but also its viability.

\section{Discussion}

The clinical guidelines from the Infectious Disease Society of America recommends the use of ALT for catheter salvage [25]. Our study suggests that ALT is a viable strategy against biofilms generated by a clinical isolate of $S$. marcescens depending on the selected antibiotics. The response to chloramphenicol and small doses of aminoglycosides offer two possible avenues to treat $S$. marcescens contaminated catheters and reduce infections associated with this organism.

A major limitation of our study is that only one strain of S. marcescens was tested against our panel of antibiotics. To date, 8 out of 14 species of Serratia are documented to be related to human infections [1]. S. marcescens is known to be endogenously resistant to antibiotics such as colistin and cephalothin [26]. In the hospital setting

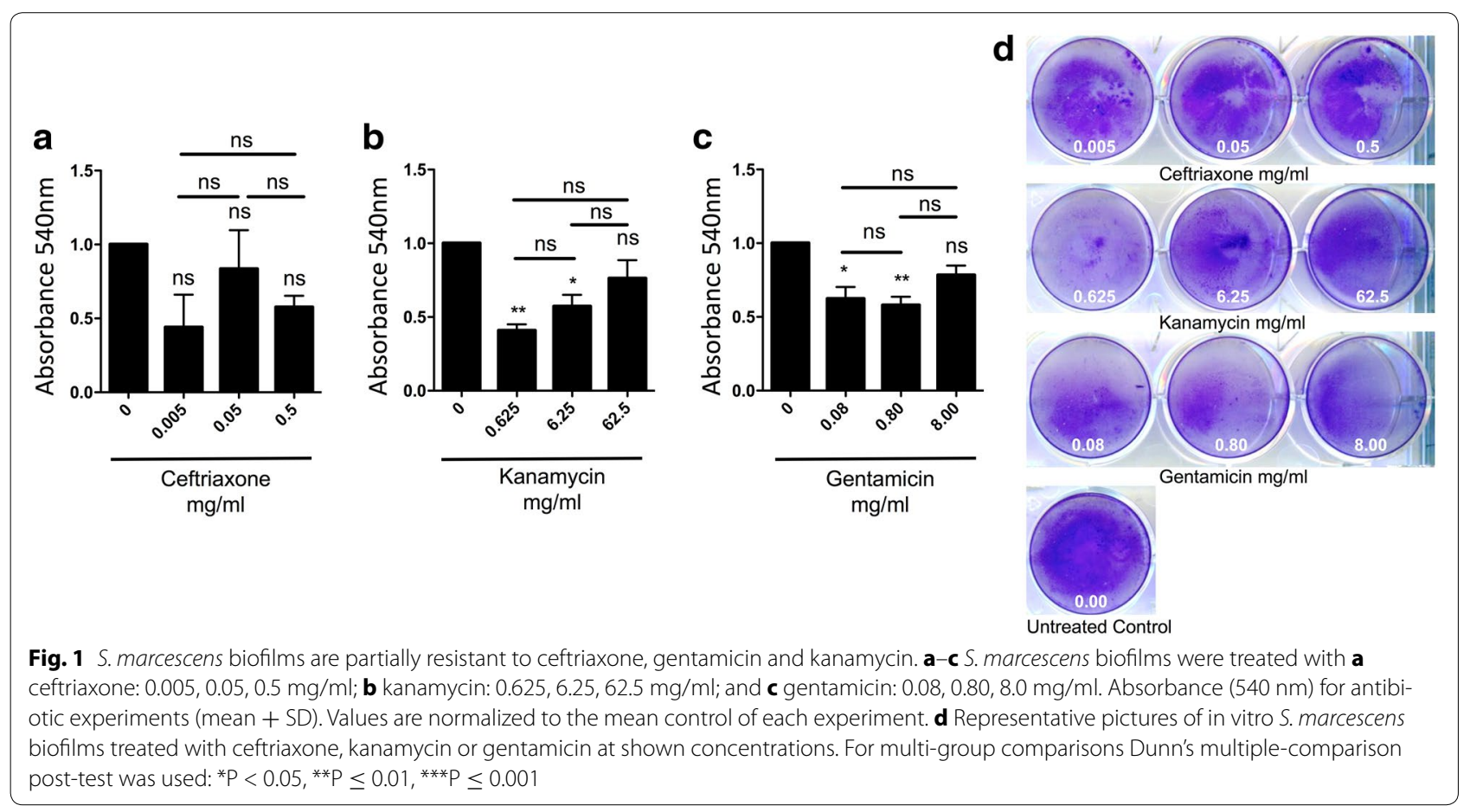




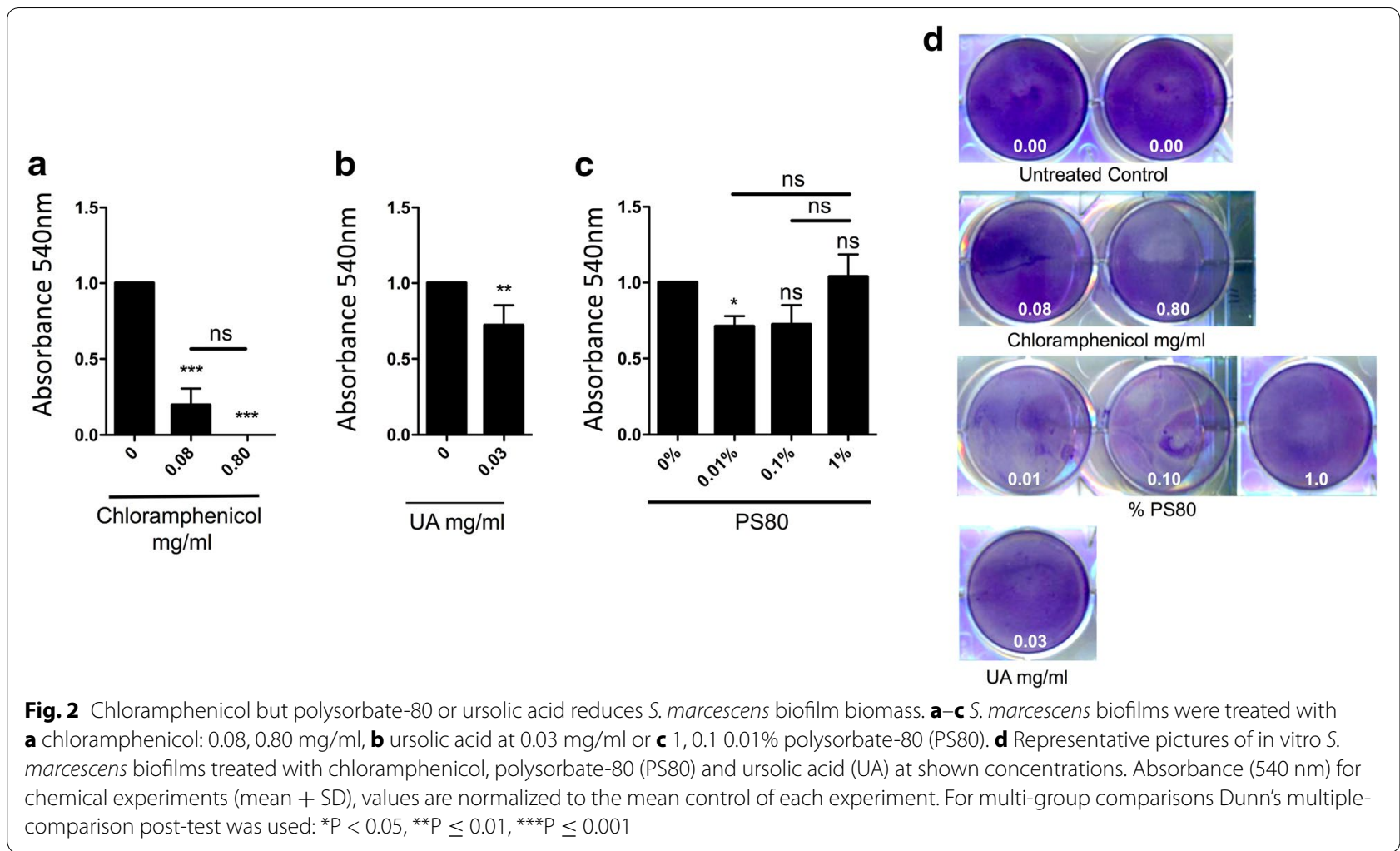

bacteria from the Enterobacteriaceae family have demonstrated to be extraordinary in their capability to acquire, transfer and express antimicrobial resistance [27]. For these reasons, another limitation of this report is that it might not represent all strains of $S$. marcescens as our clinical isolate might have gained other antimicrobial resistances in the healthcare setting not present in reference strains. Future studies are warranted to address these and other limitations. We are particularly interested in exploring the possibility that some antimicrobials might be promoting biofilm formation.

Chloramphenicol effectivity against bacterial biofilms is still not well defined [23]. A recent report found no anti-biofilm activity of chloramphenicol against Staphylococcus aureus, E. coli, and Micrococcus luteus. Importantly, the authors only used a concentration of only $0.005 \mathrm{mg} / \mathrm{ml}$, a concentration well below the planktonic MIC for our bacterium and only slightly higher than the MICs for their three isolates (maximum $0.0031 \mathrm{mg} / \mathrm{ml}$ ) [28]. In contrast, our study shows that high doses (0.08 and $0.80 \mathrm{mg} / \mathrm{ml}$ ) of chloramphenicol reduce the mass of preformed $S$. marcescens biofilms, and the $0.80 \mathrm{mg} / \mathrm{ml}$ concentration decreases the viability of biofilms compared with untreated controls. Together these results carry important implications for its potential in ALT. Importantly, since chloramphenicol is barely soluble in water, small amounts of methanol were added to facilitate solubility (yielding a final concentration of $<5 \%$ methanol in the $0.80 \mathrm{mg} / \mathrm{ml}$ solution, and $<0.5 \%$ in the $0.08 \mathrm{mg} / \mathrm{ml}$ solution); however, it seems unlikely that these small amounts of methanol influenced biofilm mass.

The use of chloramphenicol in systemic therapy has been limited by its toxicity, including fatal occurrences of granulocytopenia, aplastic anemia, and thrombocytopenia. The limitations mentioned above are avoided in ALT since the removal of the solution from the catheter minimizes systemic exposure to antibiotics [28]. The broad spectrum of action, including against some multi-drug resistant bacteria, makes chloramphenicol an attractive candidate for treating CLABSI. Additionally, chloramphenicol is inexpensive and remains stable in the presence of heparin (which is commonly included in ALT to prevent catheter thrombosis). Further studies will be needed to examine the potential of chloramphenicol as a clinical treatment with other pathogens.

\section{Conclusions}

In our study, biofilms formed by a clinical isolate of $S$. marcescens were only killed by the use of chloramphenicol at ten and one hundred times concentrations used to kill planktonic bacteria, non-other of the antibiotics tested had the same effect. These suggest that chloramphenicol might be utilized for ALT against not only 
a

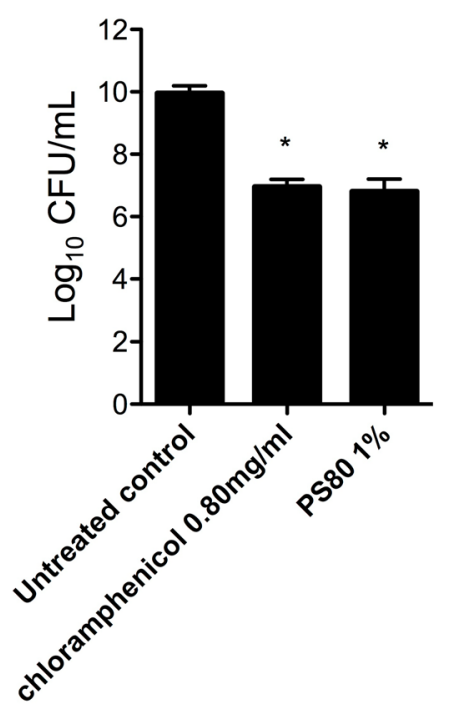

b

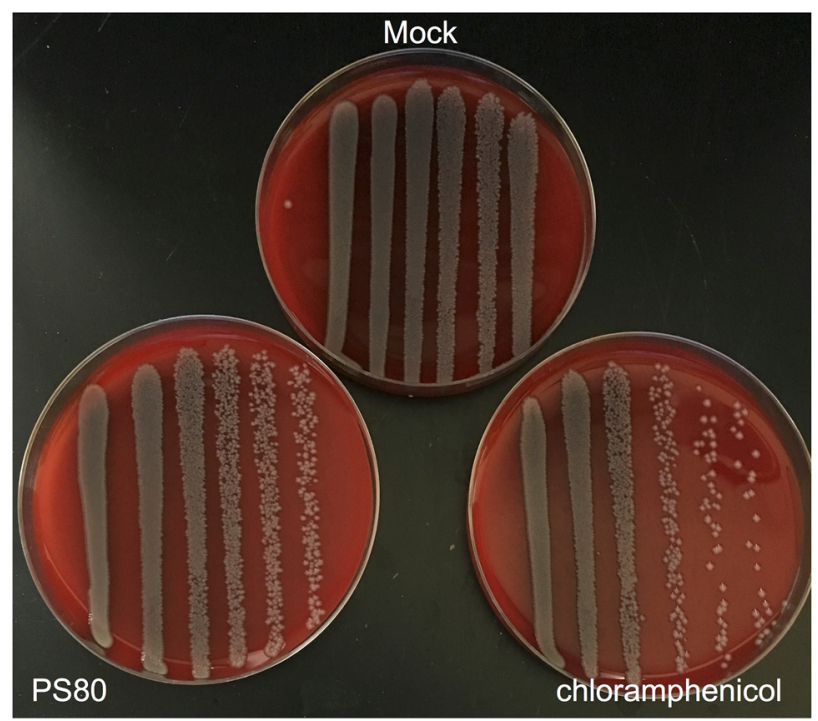

Fig. 3 Polysorbate-80 and chloramphenicol reduce biofilm viability. a Log ${ }_{10}$ CFU of S. marcescens recovered after chloramphenicol $(0.80 \mathrm{mg} / \mathrm{ml})$ or polysorbate-80 (PS80, 1\%) treatment of biofilms. b Representative images of S. marcescens recovered after chloramphenicol or polysorbate-80 treatment of biofilms. Top-untreated control; Bottom left-PS80; Bottom right-chloramphenicol. Chloramphenicol $(0.80 \mathrm{mg} / \mathrm{ml})$ and PS80: P $<0.05$. For multi-group comparisons Dunn's multiple-comparison post-test was used: ${ }^{* P}<0.05,{ }^{*} \mathrm{P} \leq 0.01,{ }^{* *} \mathrm{P} \leq 0.001$

S. marcescens but also other Gram-negative nosocomial pathogens.

\section{Additional files}

Additional file 1: Table S1. Planktonic minimum inhibitory concentration for S. marcescens $(\mathrm{mg} / \mathrm{mL})$.

Additional file 2: Table S2. Chemical Concentrations Tested.

\section{Abbreviations}

MIC: minimum inhibitory concentration; LB: Luria-Bertani media; PS80: polysorbate-80; UA: ursolic acid; CLABSI: central line-associated bloodstream infection; ALT: antibiotic lock therapy; OD: optical density; MH: Mueller-Hinton broth.

\section{Authors' contributions}

$\mathrm{NGJ}, \mathrm{CR}$, and CJO wrote and edited the paper. NGJ and CR designed the experiments. CR and ATS executed the experiments. All authors read and approved the final manuscript.

\section{Acknowledgements}

Not applicable.

\section{Competing interests}

The authors declare that they have no competing interests.

\section{Availability of data and materials}

All data supporting the conclusions of the manuscript are in Figs. 1, 2 and 3.

\section{Funding}

N.G.J. was supported by National Institutes for Health Immunologic Diseases and Basic Immunology Grant 5T32Al007051-38. C.J.O. was funded by National Institutes for Health Grant Al114800.
Received: 22 November 2016 Accepted: 17 March 2017 Published online: 29 March 2017

\section{References}

1. Mahlen SD. Serratia infections: from military experiments to current practice. Clin Microbiol Rev. 2011;24(4):755-91.

2. Gonzalez-Juarbe N, Mares CA, Hinojosa CA, Medina JL, Cantwell A, Dube $\mathrm{PH}$, Orihuela CJ, Bergman MA. Requirement for Serratia marcescens cytolysin in a murine model of hemorrhagic pneumonia. Infect Immun. 2015;83(2):614-24.

3. Bukhari SZ, Banjar A, Baghdadi SS, Baltow BA, Ashshi AM, Hussain WM Central line associated blood stream infection rate after intervention and comparing outcome with national healthcare safety network and international nosocomial infection control consortium data. Ann Med Health Sci Res. 2014;4(5):682-6.

4. Larson EL, Cimiotti JP, Haas J, Nesin M, Allen A, Della-Latta P, Saiman L. Gram-negative bacilli associated with catheter-associated and non-catheter-associated bloodstream infections and hand carriage by healthcare workers in neonatal intensive care units. Pediatr Crit Care Med. 2005;6(4):457-61

5. Sievert DM, Ricks P, Edwards JR, Schneider A, Patel J, Srinivasan A, Kallen A, Limbago B, Fridkin S, National Healthcare Safety Network, et al. Antimicrobial-resistant pathogens associated with healthcare-associated infections: summary of data reported to the National Healthcare Safety Network at the Centers for Disease Control and Prevention, 2009-2010. Infect Control Hosp Epidemiol. 2013;34(1):1-14.

6. Apostolopoulou E, Zikos D, Tselebis A, Drosatou X, Stefanidis E, Xristodoulou A, Katsaris G. Clinical outcomes and economic variables in critically ill patients with bloodstream infections. Health Sci J. 2014;8(4):519-30,

7. Satpathy S, Sen SK, Pattanaik S, Raut S. Review on bacterial biofilm: an universal cause of contamination. Biocatal Agric Biotechnol. 2016;7:56-66.

8. Flemming H-C, Wingender J, Szewzyk U, Steinberg P, Rice SA, Kjelleberg S. Biofilms: an emergent form of bacterial life. Nat Rev Micro. 2016;14(9):563-75. 
9. Nandre RM, Chaudhari AA, Matsuda K, Lee JH. Immunogenicity of a Salmonella enteritidis mutant as vaccine candidate and its protective efficacy against salmonellosis in chickens. Vet Immunol Immunopathol. 2011;144(3-4):299-311.

10. Nandre RM, Matsuda K, Chaudhari AA, Kim B, Lee JH. A genetically engineered derivative of Salmonella enteritidis as a novel live vaccine candidate for salmonellosis in chickens. Res Vet Sci. 2012;93(2):596-603.

11. Srinivasan R, Devi KR, Kannappan A, Pandian SK, Ravi AV. Piper betle and its bioactive metabolite phytol mitigates quorum sensing mediated virulence factors and biofilm of nosocomial pathogen Serratia marcescens in vitro. J Ethnopharmacol. 2016:193:592-603.

12. Donlan RM. Biofilm formation: a clinically relevant microbiological process. Clin Infect Dis. 2001;33(8):1387-92.

13. Justo JA, Bookstaver PB. Antibiotic lock therapy: review of technique and logistical challenges. Infect Drug Resist. 2014;7:343-63.

14. Snaterse M, Rüger W, Scholte op Reimer WJM, Lucas C. Antibiotic-based catheter lock solutions for prevention of catheter-related bloodstream infection: a systematic review of randomised controlled trials. J Hosp Infect. 2010;75(1):1-11.

15. Coors EA, Seybold H, Merk HF, Mahler V. Polysorbate 80 in medical products and nonimmunologic anaphylactoid reactions. Ann Allergy Asthma Immunol. 2005:95(6):593-9.

16. Malinowski AM, McClarty BM, Robinson C, Spear W, Sanchez M, Sparkes TC, Brooke JS. Polysorbate 80 and polymyxin B inhibit Stenotrophomonas maltophilia biofilm. Diagn Microbiol Infect Dis. 2017;87(2):154-6.

17. Sloup RE, Cieza RJ, Needle DB, Abramovitch RB, Torres AG, Waters CM. Polysorbates prevent biofilm formation and pathogenesis of Escherichia coli O104:H4. Biofouling. 2016;32(9):1131-40.

18. Chen H, Gao Y, Wang A, Zhou X, Zheng Y, Zhou J. Evolution in medicinal chemistry of ursolic acid derivatives as anticancer agents. Eur J Med Chem. 2015;92:648-55.
19. Zhou L, Ding Y, Chen W, Zhang P, Chen Y, Lv X. The in vitro study of ursolic acid and oleanolic acid inhibiting cariogenic microorganisms as well as biofilm. Oral Dis. 2013;19(5):494-500.

20. Gonzalez-Juarbe N, Gilley RP, Hinojosa CA, Bradley KM, Kamei A, Gao G, Dube PH, Bergman MA, Orihuela CJ. Pore-forming toxins induce macrophage necroptosis during acute bacterial pneumonia. PLoS Pathog. 2015;11(12):e1005337.

21. Iwanaga M, Toma C, Miyazato T, Insisiengmay S, Nakasone N, Ehara M. Antibiotic resistance conferred by a class I integron and SXT constin in Vibrio cholerae $\mathrm{O} 1$ strains isolated in Laos. Antimicrob Agents Chemother. 2004;48(7):2364-9.

22. Carpenter J, Stapleton S, Holliman R. Brain Abscess. Eur J Clin Microbiol Infec Dis. 2007;26:1-11.

23. Howard J. Chloramphenicol_A review. Pediatr Rev. 2004;25:284-8.

24. Rehman Au, Rehman T, Ali R. Multi-antibiotic resistant brain abscess sensitive only to chloramphenicol: a case report. Cases J. 2009;2(1):6352.

25. O'Grady NP, Alexander M, Burns LA, Dellinger EP, Garland J, Heard SO, Lipsett PA, Masur H, Mermel LA, Pearson ML, et al. Guidelines for the prevention of intravascular catheter-related infections. Am J Infect Control. 2011;39(4 Suppl 1):S1-34.

26. Matsumura N, Minami S, Mitsuhashi S. Sequences of homologous $\beta$-lactamases from clinical isolates of Serratia marcescens with different substrate specificities. Antimicrob Agents Chemother. 1998;42(1):176-9.

27. Livermore DM. Beta-lactamase-mediated resistance and opportunities for its control. J Antimicrob Chemother. 1998;41(Suppl 4):25-41.

28. Quinn GA, Maloy AP, Banat MM, Banat IM. A comparison of effects of broad-spectrum antibiotics and biosurfactants on established bacterial biofilms. Curr Microbiol. 2013;67(5):614-23.

\section{Submit your next manuscript to BioMed Central and we will help you at every step:}

- We accept pre-submission inquiries

- Our selector tool helps you to find the most relevant journal

- We provide round the clock customer support

- Convenient online submission

- Thorough peer review

- Inclusion in PubMed and all major indexing services

- Maximum visibility for your research

Submit your manuscript at www.biomedcentral.com/submit

(OioMed Central 